\title{
Intranasal Oxytocin Normalizes Amygdala Functional Connectivity in Posttraumatic Stress Disorder
}

\author{
Saskia BJ Koch*, ${ }^{*, 2}$, Mirjam van Zuiden', Laura Nawijn ${ }^{1,2}$, Jessie L Frijling ${ }^{1,2}$, Dick J Veltman ${ }^{3}$ and \\ Miranda Olff ${ }^{1,4}$
}

'Department of Psychiatry, Academic Medical Center, University of Amsterdam, Amsterdam, The Netherlands; ${ }^{2}$ Brain Imaging Center, Academic Medical Center, Amsterdam, The Netherlands; ${ }^{3}$ Department of Psychiatry, VU University Medical Center, Amsterdam, The Netherlands;

${ }^{4}$ Arq Psychotrauma Expert Center, Diemen, The Netherlands

\begin{abstract}
The neuropeptide oxytocin (OT) has been suggested as a promising pharmacological agent for medication-enhanced psychotherapy in posttraumatic stress disorder (PTSD) because of its anxiolytic and prosocial properties. We therefore investigated the behavioral and neurobiological effects of a single intranasal OT administration (40 IU) in PTSD patients. We conducted a randomized, placebo-controlled, cross-over resting-state fMRI study in male and female police officers with ( $n=37,21$ males) and without PTSD ( $n=40,20$ males). We investigated OT administration effects on subjective anxiety and functional connectivity of basolateral (BLA) and centromedial (CeM) amygdala subregions with prefrontal and salience processing areas. In PTSD patients, OT administration resulted in decreased subjective anxiety and nervousness. Under placebo, male PTSD patients showed diminished right CeM to left ventromedial prefrontal cortex (vmPFC) connectivity compared with male trauma-exposed controls, which was reinstated after OT administration. Additionally, female PTSD patients showed enhanced right BLA to bilateral dorsal anterior cingulate cortex (dACC) connectivity compared with female trauma-exposed controls, which was dampened after OT administration. Although caution is warranted, our findings tentatively suggest that OT has the potential to diminish anxiety and fear expression of the amygdala in PTSD, either via increased control of the vmPFC over the CeM (males) or via decreased salience processing of the AACC and BLA (females). Our findings add to accumulating evidence that OT administration could potentially enhance treatment response in PTSD.

Neuropsychopharmacology (20 I6) 4I, 204I-205I; doi: I0.1038/npp.20 I6. I; published online I0 February 2016
\end{abstract}

\section{INTRODUCTION}

Posttraumatic stress disorder (PTSD) develops in about $10 \%$ of trauma-exposed individuals (de Vries and Olff, 2009). Its symptoms include intrusions, avoidance of reminders of the traumatic event, negative alterations in cognitions and mood, and hyperarousal (American Psychiatric Association, 2013). Neurobiologically, PTSD is associated with amygdala hyperactivity toward negative emotional stimuli (Hayes et al, 2012) and increased activity in and connectivity between nodes of the salience network (ie, amygdala, insula, and dorsal anterior cingulate cortex (dACC) (Patel et al, 2012; Sripada et al, 2012b). Additionally, PTSD is associated with ventromedial prefrontal cortex (vmPFC) hypoactivity, as well as with decreased functional and structural connectivity between the amygdala and vmPFC (Hayes et al, 2012; Sripada et al, 2012a), supposedly leading to decreased topdown prefrontal control over the fear response.

*Correspondence: SBJ Koch, Department of Psychiatry, Academic Medical Center, University of Amsterdam, Meibergdreef 5, Amsterdam I I05 AZ, The Netherlands, Tel: +3| 2089 |3667, Fax: +3| 2089 | 3664, E-mail: s.b.koch@amc.uva.nl

Received 22 July 2015; revised 17 December 2015; accepted 24 December 2015; accepted article preview online 7 January 2016
As approximately one-third of PTSD patients completing treatment still meet criteria for PTSD (Bradley et al, 2005), there is a need for new or improved interventions to boost treatment response (de Kleine et al, 2013). Because of its anxiolytic and prosocial properties, the neuropeptide oxytocin (OT) may be a promising agent for medication-enhanced psychotherapy in PTSD (Koch et al, 2014; Olff et al, 2010). Previously, decreased amygdala reactivity toward emotional stimuli was found after intranasal OT administration in healthy males (Kirsch et al, 2005), females with borderline personality disorder (BPD) (Bertsch et al, 2013), males with generalized social anxiety disorder (GSAD) (Labuschagne et al, 2010), and male and female PTSD patients (Koch et al, 2015). In addition, OT administration increased resting-state functional connectivity between the amygdala and vmPFC in healthy males (Sripada et al, 2013) and in males with GSAD, normalizing the diminished functional connectivity observed under placebo in GSAD patients (Dodhia et al, 2014). Notably, higher amygdala reactivity in PTSD patients before treatment predicted worse treatment outcome, possibly due to (more) impaired extinction learning and fear regulation (Bryant et al, 2008b). Additionally, vmPFC-amygdala connectivity is important in fear extinction learning (Milad et al, 2007), assumed to be the underlying mechanism of 
exposure therapy in PTSD (Rothbaum and Davis, 2003). Therefore, by dampening excessive fear processing of the amygdala and increasing vmPFC to amygdala connectivity, OT is hypothesized to enhance treatment response in PTSD.

Although most neuroimaging studies conceptualize the amygdala as a single brain region, neuroanatomical studies in primates (Stefanacci and Amaral, 2002) and humans (Amunts et al, 2005) revealed that the amygdala consists of structurally and functionally heterogeneous subregions, two of which are the basolateral nucleus (BLA) and the centromedial nucleus (CeM). The BLA is involved in fear learning, by integrating information from the (somato) sensory cortex, thalamus, and dACC (Jovanovic and Ressler, 2010). The dACC has excitatory projections to the BLA, which may excite the CeM. The CeM is primarily involved in fear expression, via efferent projections to the brainstem and hypothalamus (LeDoux, 1998). In addition, the vmPFC exerts top-down inhibitory control over the CeM (Jovanovic and Ressler, 2010), via excitatory projections to GABA-ergic intercalated interneurons, which can inhibit $\mathrm{CeM}$ output. Functional imaging studies in healthy individuals have confirmed these distinct connectivity patterns (Brown et al, 2014; Roy et al, 2009).

Recently, the distinct functional connectivity patterns of the BLA and CeM were investigated in male PTSD patients, showing increased connectivity of the BLA with the pregenual anterior cingulate cortex, dACC, and dorsomedial prefrontal cortex (dmPFC) and decreased connectivity of the BLA with the inferior frontal gyrus (IFG), compared with trauma-exposed controls (Brown et al, 2014). However, when the two subregions were combined into one amygdala seed, only differential amygdala connectivity with the anterior medial PFC was found in PTSD patients (Brown et al, 2014). These findings indicate increased BLA connectivity with fear processing areas in PTSD, which would not have been identified using the entire amygdala as one seed region.

To investigate the neurobiological effects of OT administration in PTSD patients, we conducted a randomized, placebo-controlled, cross-over resting-state fMRI study in trauma-exposed police officers with and without PTSD. We specifically investigated the effects of a single intranasal OT administration on subjective anxiety and functional connectivity of BLA and CeM amygdala subregions with prefrontal areas (vmPFC) and salience processing areas (insula and dACC). We expected to find decreased connectivity between these amygdala subregions and prefrontal cortices but increased connectivity with salience network nodes in PTSD patients compared with traumaexposed controls. Additionally, we hypothesized that OT would normalize aberrant amygdala connectivity patterns in PTSD patients. We included both sexes to investigate possible sex differential effects of OT administration. This may be relevant as amygdala reactivity toward socially and non-socially threatening scenes was enhanced after OT administration in healthy females (Lischke et al, 2012), in contrast to the amygdala-dampening effects of OT administration observed in healthy males in response to comparable stimuli (Kirsch et al, 2005).

\section{METHODS}

\section{Participants}

Participants $(n=77)$ were recruited via advertisements in journals and on websites of the Dutch police and via a psychotrauma diagnostic outpatient clinic for police personnel (PDC politiepoli, Diemen, the Netherlands; PTSD patients only). Participants were between 18 and 65 years, did not have a history of neurological disorders or any current severe or chronic disease, did not use psychotropic medication, and female participants were not pregnant or breastfeeding.

Male $(n=21)$ and female $(n=16)$ PTSD patients fulfilled DSM-IV criteria for PTSD, with a score of $>45$ on the Clinician Administered PTSD Scale (CAPS; Blake et al, 1995). Current comorbidity was assessed with the Mini International Neuropsychiatric Interview (MINI-plus; Sheehan et al, 1998) or the Structured Clinical Interview for DSM-IV (First et al, 2012) (for patients recruited via the police outpatient clinic, $n=15)$. PTSD patients were excluded if they met diagnostic criteria for current substance-related disorder, severe major depressive disorder (MDD), psychotic disorder, personality disorder, or current suicidal risk.

Forty trauma-exposed male $(n=20)$ and female $(n=20)$ controls were matched to the PTSD patients on sex, age, years of service, and educational level. Control participants were exposed to at least one potentially traumatic event, according to the DSM-IV A1 PTSD criterion, and had a CAPS score of $\leqslant 15$. Trauma-exposed controls did not have any current DSM-IV axis-1 psychopathology and no history of PTSD or MDD, as assessed with the MINI-plus.

The study was conducted in accordance with the declaration of Helsinki and approved by the Institutional Review Board of the Academic Medical Center in Amsterdam, The Netherlands. All participants provided written informed consent before study participation.

\section{Experimental Procedure}

This within-subjects study consisted of three appointments: one baseline session (T0) and two fMRI sessions (T1 and T2). During T0, inclusion and exclusion criteria were assessed. T1 and T2 were scheduled on average 11.5 $( \pm 9.90)$ days apart and preferably took place in the afternoon or evening. Participants were asked to abstain from alcohol and drugs $24 \mathrm{~h}$ before scanning and from food, nicotine, beverages (except water), and rigorous exercise $2.5 \mathrm{~h}$ prior to scanning. Before scanning, participants self-administered intranasal OT (40 IU, Syntocinon, five puffs of $4 \mathrm{IU}$ per nostril) during one fMRI session and placebo (saline, $\mathrm{NaCl}$ $0.9 \%$, five puffs per nostril) during the other fMRI session, under experimenter supervision. The dose of $40 \mathrm{IU}$ was based on a previous study in which therapeutic effects of OT administration in psychiatric patients were observed (Feifel et al, 2010). The order of nasal spray administration was randomized, double-blind, and counter-balanced between sessions. The resting-state scan was part of a larger scanning protocol. First, two task-based fMRI scans were conducted: an emotional face-matching task (Koch et al, 2015) and a monetary (Nawijn et al, 2016a) and social (Nawijn et al, 2016b) incentive delay reward task. Hereafter, the resting- 
state scan was conducted, which began on average $72.51 \mathrm{~min}$ $( \pm 4.03)$ after intranasal spray administration, when neuropharmacological OT administration effects on cerebral blood flow (Paloyelis et al, 2014) and elevated OT levels in cerebrospinal fluid (Striepens et al, 2013) are still observed. During resting-state scanning, participants were instructed to relax and let their mind wander, with open eyes. At the end of each scanning session, we assessed whether participants had let their mind wander during resting-state scanning (yes-no question). In case specific thoughts were reported, content of thought was assessed with an open question. To investigate behavioral effects of OT administration, we assessed current levels of anxiety, nervousness, happiness, and sadness on visual analog scales (VAS) from 0 (not at all) to 100 (entirely). During each fMRI session, these VAS questionnaires were administered before intranasal spray administration and after functional scanning was completed. Finally, after each scanning session, participants were asked to guess which intranasal spray they received and the primary reason for their guess.

\section{fMRI Acquisition}

Scanning was performed with a 3-T Philips Achieva MR system (Philips Medical Systems, Best, The Netherlands), using a 32-channel head coil. A FAST MPRage sequence was used to obtain a high-resolution anatomical scan (220 slices; voxel size $=1 \mathrm{~mm}^{3}$; repetition time $=8.2 \mathrm{~s}$; echo time $=3.8 \mathrm{~s}$; flip angle $=8^{\circ}$ ). Resting-state scanning was performed using an echo planar sequence sensitive to BOLD contrast ( $7.9 \mathrm{~min} ; 238$ volumes; 37 slices; voxel size $=3 \mathrm{~mm}^{3}$; repetition time $=2 \mathrm{~s}$, echo time $=28 \mathrm{~ms}$; flip angle $=76^{\circ}$ ).

\section{Data Analysis}

Demographics and questionnaires. SPSS version 20 (IBM Statistics, Armonk, NY, USA) was used to assess differences in demographical characteristics between PTSD patients and trauma-exposed controls, for male and female participants separately. After checking for outliers and normality and (log)transforming when necessary, independent $t$-tests and repeated-measures ANOVAs were performed for continuous variables and chi-square tests for categorical variables. Additionally, we used two repeated-measures ANOVAs to compare subjective anxiety and mood ratings during the OT and placebo sessions: one for ratings acquired before drug administration and one for ratings acquired after scanning. Drug order was included as covariate. VAS ratings were missing for two male PTSD patients. A $P$-value $<0.05$ (twotailed) was considered significant.

\section{fMRI Data Analysis}

SPM8 (http://www.fil.ion.ucl.ac.uk/spm) was used for fMRI analysis. Amygdala seeds for the left and right BLA and CeM subregions were defined using the Juelich histological atlas (Amunts et al, 2005; Eickhoff et al, 2005) as implemented in FSL (FMRIB Software Library, http://fsl.fmrib.ox.ac.uk/fsl; see Figure 1a). For the CeM, voxels were included if they had $a \geqslant 50 \%$ probability of belonging to the CeM amygdala subregion according to the Juelich atlas. Because of signal drop-out in the temporal cortex, a more stringent probability threshold of $80 \%$ (and hence a smaller seed) was used for the BLA subregion. For each participant, the first eigenvariates of the left and right BLA and CeM amygdala time courses were extracted using the Volume of Interest module in SPM8, which were subsequently used as regressor of interest in the first-level models (see Supplementary Material pages 2-3 for details regarding preprocessing and first-level analyses).

Contrast estimates of positive correlations with the amygdala seeds were used in second-level repeated-measures ANOVAs, for each left and right-sided amygdala seed separately. Between-subjects factors included group (PTSD-control) and sex (male-female) and the withinsubjects factor included drug (OT-placebo). Drug order
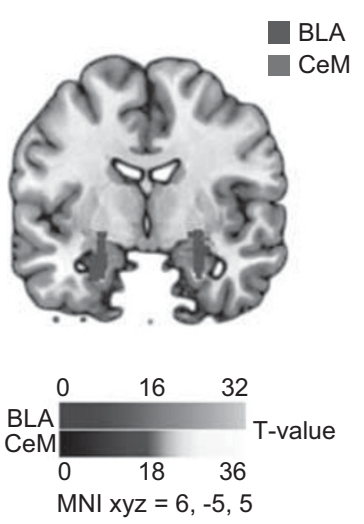

b

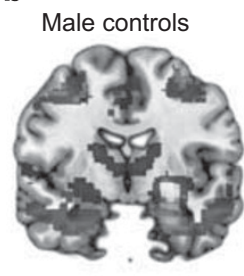

C

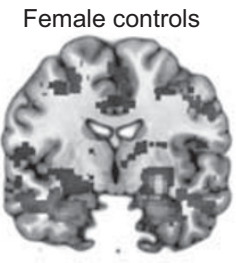

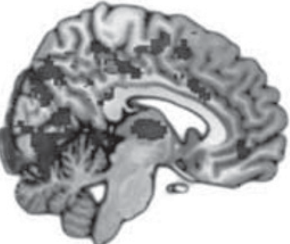

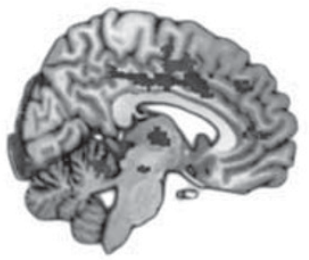

d

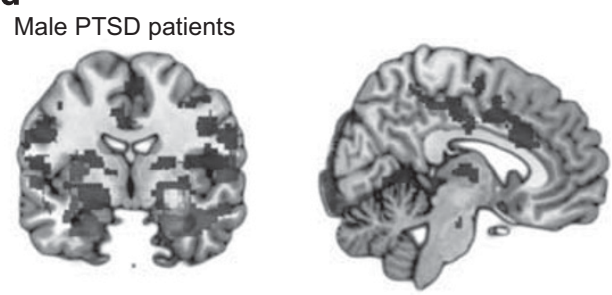

e Female PTSD patients
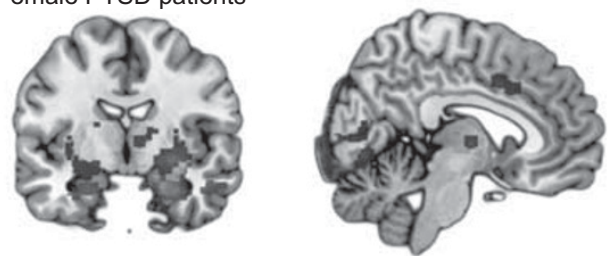

Figure I BLA and CeM subregion connectivity. (a) Seeds for the basolateral (BLA) and centromedial (CeM) amygdala subregions used in the functional connectivity analyses, defined using the Juelich probabilistic atlas (Amunts et al, 2005; Eickhoff et al, 2005). Right-sided BLA and CeM connectivity under placebo in (b) male trauma-exposed controls, (c) female trauma-exposed controls, (d) male PTSD patients, and (e) female PTSD patients. Blue overlay represents the right-BLA functional connectivity pattern; red overlay represents the right CeM functional connectivity pattern $(M N I x, y, z=6,-5,5)$. Left and right-sided connectivity patterns were comparable. A full color version of this figure is available at the Neuropsychopharmacology journal online. 
was added as a covariate. Additionally, we calculated the mean frame-wise displacement (FD) for each individual, for each session, using the Data Processing Assistant Resting-State fMRI toolbox (Chao-Gan and Yu-Feng, 2010). Mean FD values were added as a covariate to all second-level models, to account for variance in relative displacement between scans (Yan et al, 2013).

We tested within predefined regions of interest (ROIs) created with the anatomical 50\% Harvard-Oxford probabilistic atlas (http://fsl.fmrib.ox.ac.uk/fsl/fslwiki/Atlases) corresponding to these regions: insula, vmPFC, (ie, subcallosal ACC mask), dmPFC (ie, superior frontal gyrus mask), middle frontal gyrus, orbital frontal cortex (OFC), and ACC (ie, ACC and paracingulate cortex masks). $P$-values were family-wise error (FWE) corrected for multiple comparisons within predefined ROIs using small volume correction. For significant interaction effects $\left(P_{\mathrm{FWE}}<0.05\right)$, contrast estimates were extracted using Marsbar (http://marsbar.source forge.net) with a 5-mm sphere surrounding the peak voxel of the interaction effects and post hoc tested in SPSS. Alphavalues were Bonferroni corrected for the number of post-hoc $t$-tests conducted for each ROI (eg, $0.05 / 8=0.0063$ ).

Two trauma-exposed controls were excluded from the analyses owing to scanner artifacts. Additionally, two male and two female PTSD patient were excluded owing to excessive movement ( $>6 \mathrm{~mm} /$ degrees in any direction). After exclusion of these participants, average translation and rotation in any direction were not significantly different between PTSD patients and trauma-exposed controls (all $P>0.05)$. In total, 33 PTSD patients (19 males) and 38 trauma-exposed controls (19 males) were included in CeM subregion analyses. For BLA amygdala subregion analyses, additional participants had to be excluded because of signal drop-out in voxels belonging to these seeds (one participant for left and two participants for right BLA, see Supplementary Methods). To ensure that our analyses were not driven by excessive head motion, we reran the analyses with a more conservative threshold of $3 \mathrm{~mm} /$ degrees in any direction, additionally excluding one female participant, two male, and two female PTSD patients (see Supplementary Methods).

\section{RESULTS}

\section{Demographics}

PTSD patients did not differ from trauma-exposed controls regarding age, years of service, educational level, hormonal contraceptive use (females only), and time between intranasal spray administration and resting-state scanning (all $P>0.05$; see Table 1). Female trauma-exposed controls experienced more types of work-related traumatic events compared with female PTSD patients $(P=0.042)$, whereas male PTSD patients experienced more types of childhood traumatic events than male trauma-exposed controls $(P=0.037)$. Of all participants, $85.91 \%$ reported alternating thoughts during resting-state scanning, as opposed to thoughts about a specific topic (ie, 14.09\%). Both PTSD patients and trauma-exposed controls were equally successful at letting their mind wander during resting-state scanning (T1 controls: $89.74 \%$ alternating thoughts, PTSD: $80.56 \%$ alternating thoughts, $P=0.264$; T2 controls: $89.74 \%$ alternating thoughts, PTSD: $82.86 \%$ alternating thoughts, $P=0.390$ ). During each scanning session, three PTSD patients reported symptom-related thoughts during restingstate scanning, whereas three trauma-exposed controls reported thoughts about leisure activities, family, and friends (see Supplementary Table S1 for thought content in PTSD patients and trauma-exposed controls during the first and second fMRI session).

\section{Functional Connectivity-CeM Amygdala}

See Figure $1 \mathrm{~b}$ for CeM and BLA functional connectivity patterns in male and female trauma-exposed controls and PTSD patients under placebo. A significant group by sex by drug interaction effect was found regarding functional connectivity of the right CeM with the left vmPFC (peak voxel $x, y, z=-2,30,-8, \mathrm{Z}=2.86, K=16, P_{\mathrm{FWE}}=0.032$; see Figure 2). We therefore investigated OT administration effects on right CeM to left vmPFC functional connectivity separately for male and female PTSD patients and traumaexposed controls. Under placebo, male PTSD patients showed significantly less right CeM to left vmPFC connectivity compared with male trauma-exposed controls $(t(35)=3.93 ; P<0.001)$, whereas no connectivity differences were found for female participants $(P=0.890)$. In addition, we found a trend significant group by sex interaction effect regarding right $\mathrm{CeM}$ to right vmPFC connectivity (peak voxel $x, y, z=2,30,-8, Z=2.39, K=5, P_{\mathrm{FWE}}=0.077$ ), in which the male trauma-exposed controls showed stronger connectivity between the right-sided CeM and vmPFC compared with male PTSD patients $(t(35)=3.18, P=0.003)$. OT administration resulted in enhanced connectivity between the right CeM and left vmPFC in male PTSD patients $(t(17)=-3.32, P=0.004)$ but not in female PTSD patients or trauma-exposed controls (all $P>0.05$ ). The under placebo observed difference in right CeM to left vmPFC connectivity between male PTSD patients and male trauma-exposed controls was no longer significant after OT administration $(P=0.570)$. These results remained significant after additionally excluding participants moving $>3 \mathrm{~mm} /$ degrees in any direction (see Supplementary Table S2 and S3).

Controlling for differences in the number of different types of work-related and childhood traumatic events did not alter our results. No main effects of drug, group by drug, or sex by drug interactions were observed for left and right CeM functional connectivity (all $P_{\mathrm{FWE}}>0.05$ ). Under placebo, no main effects of sex or group or sex by group interactions were found regarding left and right CeM functional connectivity.

\section{Functional Connectivity-BLA Amygdala}

When investigating OT administration effects on left and right BLA functional connectivity, we found a (trend) significant group by sex by drug interaction effect regarding connectivity of the right BLA with the bilateral dACC (left: peak voxel $x, y, z=-8,50,16 ; Z=3.27, K=26, P_{\mathrm{FWE}}=0.070$; right: peak voxel $x, y, z=6,38,34 ; Z=3.70, K=99$, $\left.P_{\mathrm{FWE}}=0.019\right)$. Therefore, OT administration effects on right BLA connectivity with the bilateral dACC were investigated separately for male and female PTSD patients and trauma-exposed controls. Further testing showed that OT 
PTSD patients $(n=36)$

Males $(n=21)$

Females $(n=16)$

$42.29(9.83)$

I $6.29(10.82)$

Years of service

Educational level

Low

Middle

High

CAPS total score (PTSD severity)

Current comorbidity (N)

MDD

Dysthymia

Panic disorder

Specific phobia

PLES, number of different work-related traumatic experiences

ETI, number of different childhood traumatic experiences

Hormonal contraceptive use

No

Menopause

$\begin{array}{cc}0(0 \%) & 0(0 \%) \\ 17(81 \%) & 15(93 \%) \\ 4(19 \%) & 1(7 \%)\end{array}$

$68.05(15.62)$

$67.56(11.83)$

$4(25 \%)$

I (6.3\%)

$2(9.5 \%)$

I (4.8\%)

I (4.8\%)

$22.50(5.95)$

$6.09(4.55)$

$13.50(4.49)$

$5.25(5.18)$

NA

$7(44 \%)$

$8(50 \%)$

I (6\%)

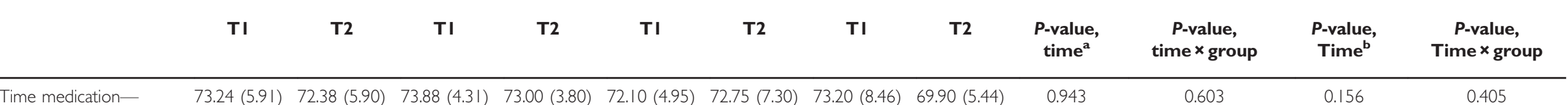

Healthy controls $(n=40)$

Group difference

Males $(n=20) \quad$ Females $(n=20)$

Males,
P-value

Females, $P$-value

P-value

$1.35(10.62)$

$38.65(9.48)$

$18.60(9.84)$

0.516

0.738

$8.42(10.05)$

$0(0 \%)$

$0(0 \%)$

$17(85 \%)$

$6(80 \%)$

$3(15 \%)$

$4(20 \%)$

$4.45(4.66)$

$<0.001$

$<0.001$

$4.70(4.79)$

NA

NA

NA

NA

.193

Time medicationresting-state ( $\mathrm{min}$ )

Abbreviations: CAPS, Clinician Administered PTSD Scale; ETI, early trauma inventory, number of different events; PLES, police life events scale; MDD, major depressive disorder; NA, not applicable.

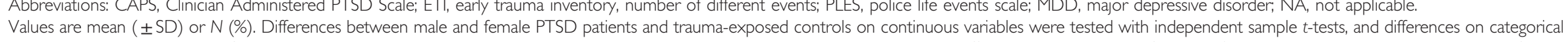

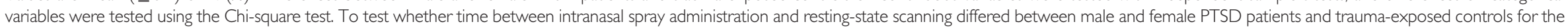
first and second fMRI session, a repeated-measures ANOVA was conducted. Significant group differences $(P<0.05)$ are printed in bold.

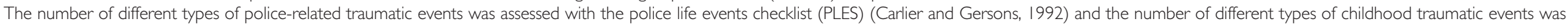
assessed with the self-report version of the Early Trauma Inventory (ETI) (Bremner et al, 2007).

aMain effect of group $(P=0.758)$

bain effect of group $(P=0.184)$ 
a

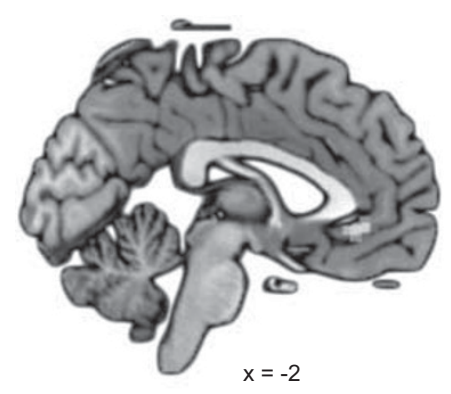

15

F-value

\section{b}

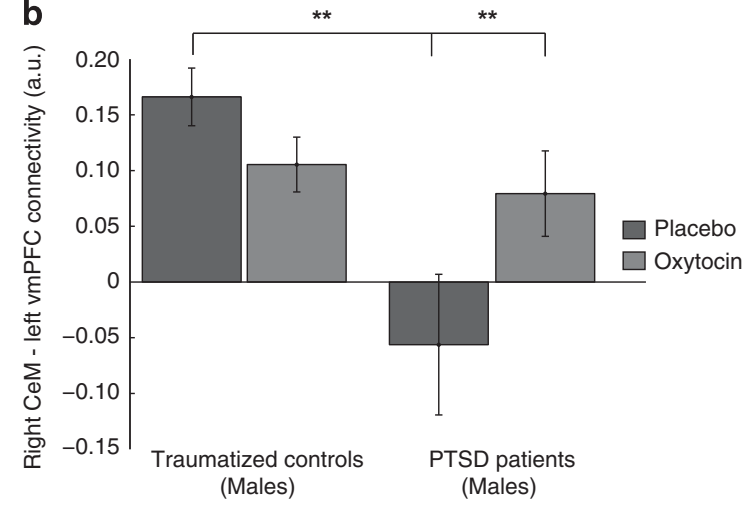

Figure 2 Right CeM amygdala-left vmPFC connectivity. (a) Statistical map of the drug by group by sex interaction effect regarding functional connectivity of the right centromedial amygdala (CeM) with the left ventromedial prefrontal cortex (vmPFC). Results are overlaid on a single-subjects template and statistically thresholded at $P<0.005$ (uncorrected) for display purposes. (b) Contrast estimates in arbitrary units (a.u.) of right CeM connectivity with the vmPFC (5 mm sphere, peak voxel $x, y, z=-2,30,-8)$ in male trauma-exposed controls and male PTSD patients. Error bars represent SEM. ** P<0.005. A full color version of this figure is available at the Neuropsychopharmacology journal online.
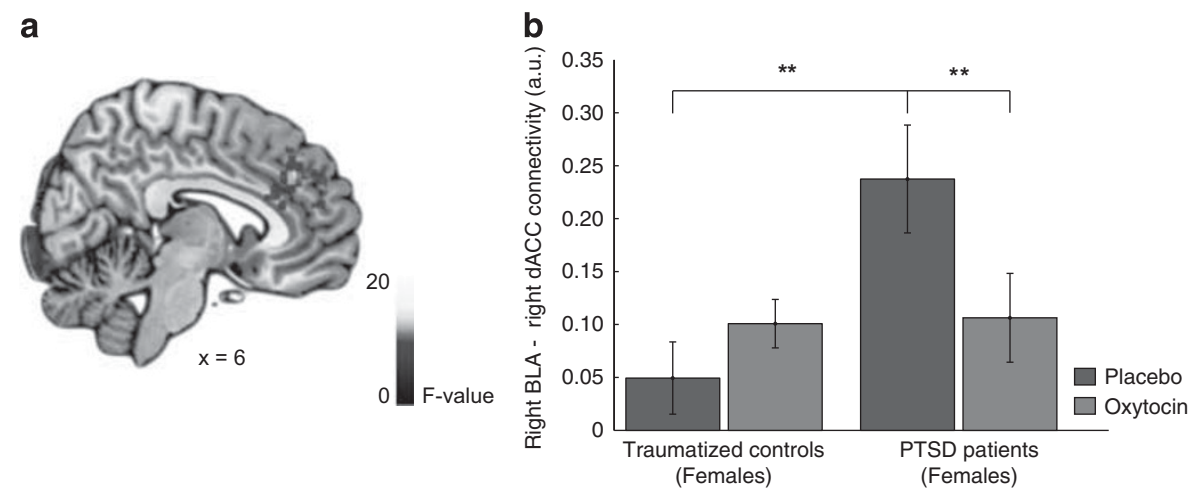

Figure 3 Right BLA amygdala-dACC connectivity. (a) Statistical map of the group by sex by drug interaction effect regarding functional connectivity of the right basolateral amygdala (BLA) with the dorsal anterior cingulate cortex (dACC). Results are overlaid on a single-subjects template and statistically thresholded at $P<0.005$ (uncorrected) for display. (b) Contrast estimates in arbitrary units (a.u.) of right BLA-right dACC (5 mm sphere, peak voxel $x, y, z=6$, $38,34)$ connectivity in female PTSD patients and trauma-exposed controls. Error bars represent SEM. $* * P<0.005$. A full color version of this figure is available at the Neuropsychopharmacology journal online.

administration decreased connectivity between the right BLA and right dACC in female PTSD patients $(t(12)=4.45$, $P=0.001$; see; Figure 3) and tended to decrease right BLA to left dACC connectivity in female PTSD patients $(t(12)=2.61$, $P=0.023)$ and in male trauma-exposed controls $(t(18)=$ $2.49, P=0.023)$. Under placebo, we found significantly greater right BLA to right dACC connectivity in female PTSD patients compared with female trauma-exposed $(t(30)=-3.19, P=0.001)$. This difference between female PTSD patients and trauma-exposed controls was absent after OT administration $(P=0.902)$. These results remained significant after more stringently excluding participants with excessive head movement (ie, $3 \mathrm{~mm} /$ degrees in any direction; see Supplementary Table S2 and S3). Regarding left BLA connectivity, no significant OT administration effects were found for PTSD patients and trauma-exposed controls (all $\left.P_{\mathrm{FWE}}>0.05\right)$. Controlling for differences in the number of different types of work-related and childhood traumatic events did not alter our results. No main effects of drug, group by drug, or sex by drug interactions were observed for left and right BLA functional connectivity (all $P_{\mathrm{FWE}}>0.05$ ). No main effects of drug or other group by drug interactions were observed for left and right BLA functional connectivity (all $P_{\mathrm{FWE}}>0.05$ ).

Under placebo, we observed a significant group by sex interaction effect for left BLA functional connectivity with the right insula (peak voxel $x, y, z=34,22,-4$; $\left.Z=4.10, K=50, P_{\mathrm{FWE}}=0.003\right)$, in which male traumaexposed controls showed enhanced functional connectivity between the left BLA and right insula compared with female trauma-exposed controls $(P=0.045)$. No sex differences were observed between male and female PTSD patients regarding left BLA-right insula connectivity $(P=0.152)$. Additionally, a significant group by sex interaction effect was observed for functional connectivity of the right BLA with the left IFG (peak voxel $x, y, z=-50,18,4 ; Z=3.69, K=29, P_{\mathrm{FWE}}=$ 0.006), in which male trauma-exposed controls showed increased functional connectivity between the right BLA and left IFG, compared with female trauma-exposed controls $(P=0.005)$. Again, no sex differences were found between male and female PTSD patients regarding right BLA to left IFG functional connectivity $(P=0.186$; see Supplementary Table S4 for test statistics of these group and sex differences under placebo). 


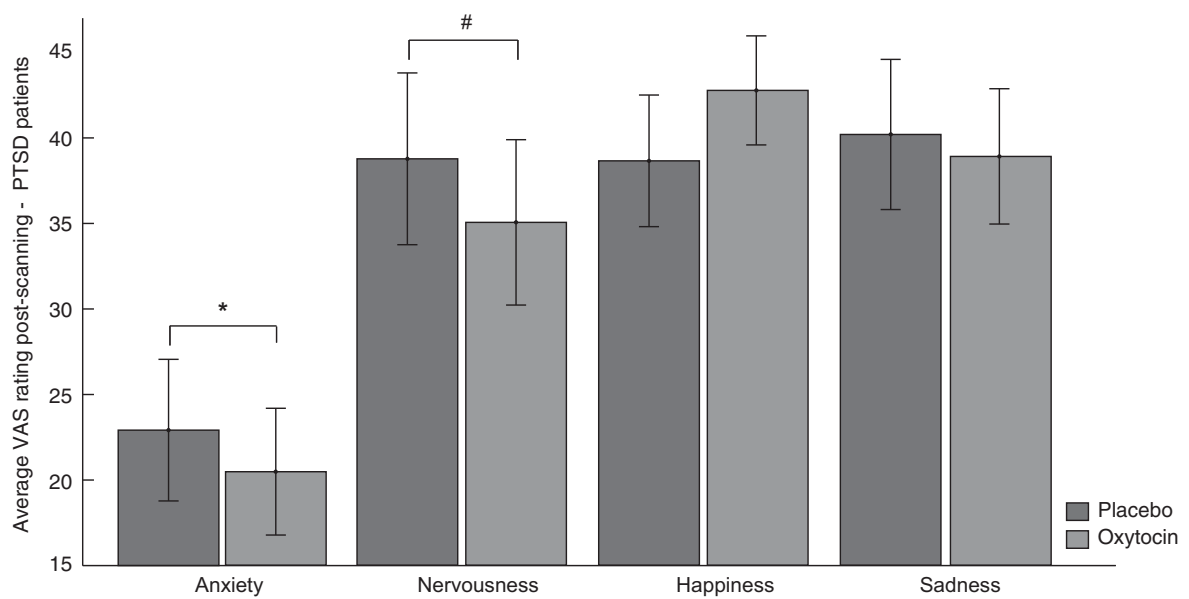

Figure 4 Behavioral effects of OT administration in PTSD patients. Average rating on visual analog scales (VAS) assessing current subjective anxiety, nervousness, happiness, and sadness on a scale of 0 (not at all) to 100 (entirely) after placebo and OT administration in PTSD patients (males and females combined). Error bars represent SEM. $* P<0.05, \# P<0.1$.

\section{Behavioral OT Effects in PTSD Patients}

We investigated whether OT administration altered subjective experiences in PTSD patients. Compared with placebo, OT administration in PTSD patients was associated with lower ratings of subjective anxiety $(F(1,32)=4.42$, $P=0.044)$ and nominally lower ratings of nervousness $(F(1,32)=3.98, P=0.055)$ but not happiness and sadness (all $P>0.05$ ) (see Figure 4; see Supplementary Figure S1 for subjective ratings in male and female PTSD patients separately). Ratings of anxiety, nervousness, happiness, and sadness prior to drug administration did not differ between scanning sessions (all $P>0.05$ ). OT-induced reductions in anxiety and nervousness were not significantly correlated with OT-induced alterations in amygdala functional connectivity with the vmPFC (male PTSD patients) or right dACC (female PTSD patients) (all $P>0.05$; Supplementary Table S5). CAPS total and subscale scores were not significantly correlated to functional connectivity measures under placebo (all $P>0.05$; Supplementary Table S6).

After the first scanning session, $51.4 \%$ of trauma-exposed controls and $57.1 \%$ of PTSD patients correctly guessed which intranasal spray they received, which is not significantly different from chance levels (ie, 50\%, binominal test, controls: $P=1.00$, PTSD: $P=0.500)$. After the second scanning session, trauma-exposed controls still guessed at chance level (ie, $62.2 \%$ of controls guessed correctly, 50\% binominal test, $P=0.188$ ), whereas PTSD patients were able to correctly guess intranasal treatment allocation (ie, $77.1 \%$ of PTSD patients guessed correctly, 50\% binominal test, $P=0.002)$. This seemed more pronounced in female PTSD patients $(86.7 \%$ correct, $50 \%$ binominal test, $P=0.007)$ than in male PTSD patients (70\% correct, $50 \%$ binominal test, $P=0.115)$. Of note, in those participants correctly guessing intranasal treatment allocation during the second scanning session, the most common reason for their correct guess was psychological effects, such as increased calmness, sleepiness, and changes in concentration (ie, 59.09\% of controls and $55.56 \%$ of patients), whereas a minority reported mild side effects (eg, dizziness, headache; ie, $4.54 \%$ of controls and $7.41 \%$ of patients) or differences in taste or smell between placebo and oxytocin (ie, $22.7 \%$ of controls and $29.6 \%$ of patients) as primary reason.

\section{DISCUSSION}

We investigated OT administration effects on amygdala subregion functional connectivity in male and female PTSD patients and trauma-exposed controls. We found diminished functional connectivity between the right $\mathrm{CeM}$ and left vmPFC in male PTSD patients under placebo, which was reinstated after OT administration. Female PTSD patients, on the other hand, showed higher connectivity between the right BLA and right dACC under placebo, which was attenuated after OT administration to levels similar to that of female trauma-exposed controls under placebo. These neural effects were paralleled by decreased subjective anxiety and nervousness in PTSD patients after OT administration.

Under placebo, male PTSD patients showed lower CeM to vmPFC connectivity compared with male trauma-exposed controls. Previously, Sripada et al (2012a) found decreased anticorrelations between amygdala and vmPFC activity in male PTSD patients compared with male traumatized controls, indicating decreased inhibitory connectivity between the vmPFC and amygdala. Decreased amygdala to prefrontal connectivity is thought to represent decreased top-down control over the fear response, via excitatory glutamatergic vmPFC projections to GABA-ergic interneurons of the amygdala, which in turn inhibit fear expression of the CeM (Jovanovic and Ressler, 2010). In line with the notion of top-down control, vmPFC activity has been positively associated with extinction learning in healthy individuals, inhibiting the conditioned fear response of the amygdala when an extinction memory is retrieved (Milad et al, 2007). Notably, we found OT-induced decreased subjective anxiety, as well as increased connectivity between the vmPFC and CeM in male PTSD patients. Based on the prevailing amygdala neurocircuitry model, increased $\mathrm{CeM}$ to vmPFC connectivity may indicate increased top-down prefrontal control over the fear response, although caution 
should be warranted in assigning causal relations to correlational resting-state data.

Our finding of stronger BLA to dACC connectivity in female PTSD patients compared with female traumaexposed controls replicates a previous finding on amygdala subregion connectivity, albeit in male veterans with PTSD (Brown et al, 2014). The dACC is implicated in the expression of fear via excitatory glutamatergic projections to the BLA, which in turn may increase fear-associated output of the CeM (Jovanovic and Ressler, 2010). Furthermore, the dACC and amygdala are key nodes of the salience network, which is important for directing attention to salient stimuli in the environment. This is particularly relevant in light of the commonly observed hypervigilance in PTSD. Indeed, dACC activity in female PTSD patients was previously associated with attentional bias toward threatening facial expressions in a dot-probe task (Fani et al, 2012). We found that OT administration attenuated BLA-dACC connectivity in female PTSD patients to the same level as female trauma-exposed controls under placebo. Reducing (excessive) connectivity between the dACC and amygdala in PTSD patients may lead to decreased hypervigilance, thereby dampening fear responsiveness and decreasing subjective anxiety and nervousness.

Behaviorally, PTSD patients reported decreased subjective anxiety and nervousness after OT administration but no alterations in self-reported happiness or sadness. This is in line with a previous study, in which reduced subjective anxiety during public speaking stress in healthy males was observed after a single OT administration (de Oliveira et al, 2012). PTSD patients, who are characterized by exaggerated fear responses, may be especially sensitive to the anxiolytic effects of OT administration, resulting in decreased reported anxiety and nervousness in this group specifically. In addition, $77.1 \%$ of PTSD patients (but not trauma-exposed controls) were able to correctly guess treatment allocation at the end of the second scanning session, reporting noticeable OT administration effects on psychological functioning as primary reason underlying their guess. These findings seem to be in line with the suggestion that OT administration effects may be especially beneficial for those individuals who have something to gain regarding fear regulation (Labuschagne et al, 2010). As mentioned previously, our observed OT-induced alterations in amygdala subregion connectivity may have resulted in decreased fear expression of the CeM, either via increased (inhibitory) top-down control of the vmPFC over the CeM (male PTSD patients) or via decreased excitation of the BLA by the AACC (female PTSD patients). This could be a potential underlying neural mechanism of the observed decreased subjective anxiety and nervousness. However, we did not find significant associations between OT-related reductions in anxiety and nervousness and OT-induced alterations in amygdala subregion functional connectivity. Several possible explanations can be put forward. First, anxiety and nervousness were assessed at the end of the fMRI session, approximately $25 \mathrm{~min}$ after resting-state scanning, and therefore we cannot exclude additional influences on these behavioral outcomes. Second, male and female PTSD patients showed differential neural, but not behavioral, correlates of OT administration. Therefore, we conducted subgroup analyses on the association between OT-induced alterations in subjective anxiety and amygdala subregion connectivity for male and female PTSD patients separately. Possibly, these analyses suffered from a lack of power owing to the subgroup analysis. This may be especially true for male PTSD patients, in whom the correlation coefficients between OT-induced subjective and neural alterations were of moderate effect size (ie, anxiety: Pearson's $r=0.333$; nervousness: Pearson's $r=0.336$ ).

In contrast to a previous intranasal fMRI resting-state study in which increased amygdala to vmPFC connectivity was found after OT administration in healthy individuals (Sripada et al, 2013), we did not find OT administration effects in our healthy trauma-exposed controls, except for the dampening of right BLA to right dACC connectivity in male controls. It has been suggested that the effects of OT depend on inter-individual and contextual factors (Bartz et al, 2011) and may be more beneficial in those who have something to gain regarding fear regulation (Labuschagne et al, 2010; Olff et al, 2013). Of note, we included a healthy sample of apparently highly resilient controls, with few PTSD symptoms and no current psychopathology, despite high trauma exposure. Presumably, they had little to gain regarding fear regulation, explaining the almost complete absence of OT administration effects in this group.

Notably, our male and female PTSD patients showed distinct amygdala functional connectivity abnormalities compared with their same-sex traumatized controls, suggesting that the underlying neurobiological correlates of PTSD differs for males and females To our knowledge, to date only one fMRI study investigated sex differences in functional connectivity of amygdala subregions, albeit in relation to the personality trait harm avoidance in healthy individuals ( $\mathrm{Li}$ et al, 2012). Harm avoidance was more strongly associated with functional connectivity patterns of the BLA in females and of the CeM in males (Li et al, 2012). However, sex differences in functional connectivity patterns of amygdala subregions have not yet been studied in PTSD patients. Our findings underline the importance of investigating such possible neurobiological sex differences in PTSD.

Presumably, the effects of OT administration on amygdala subregion connectivity in PTSD patients may be associated with dampened fear expression toward threat-related stimuli. Although causal relations cannot be inferred from these correlational data, the increased connectivity between the CeM and vmPFC after OT administration observed in male PTSD patients may suggest increased prefrontal control over the amygdala. Additionally, the decreased BLA to dACC connectivity observed in female PTSD patients may represent decreased excitatory dACC projections to the BLA. However, this remains speculative. In line with the notion of dampened fear expression, PTSD patients showed decreased anxiety and nervousness after OT, but no alterations in mood. In PTSD patients, higher amygdala reactivity toward masked fearful faces, measured before psychotherapy, has been associated with worse treatment outcome (Bryant et al, 2008a). In addition, increased amygdala and dACC activity toward pictures of negative emotional scenes before treatment predicted the persistence of PTSD symptoms posttreatment (van Rooij et al, 2014). It has been suggested that excessive fear during psychotherapy could impair extinction learning, resulting in less favorable therapy outcomes (Bryant et al, 2008a). Successful treatment has been associated with decreased amygdala activity and increased vmPFC activity 
toward emotional faces over the course of treatment (Felmingham et al, 2007). Notably, the vmPFC is important in extinction learning, the underlying mechanism of exposure therapy (Rothbaum and Davis, 2003) and the treatment of choice for PTSD (Foa et al, 2009). Previous animal (Zoicas et al, 2014) and human studies (eg, Eckstein et al, 2014) have shown that OT administration enhanced extinction learning (but see (Acheson et al, 2015). In healthy individuals, OT administration increased prefrontal involvement during the early phase of fear extinction and attenuated general amygdala responsivity (Eckstein et al, 2014). Taken together, decreasing (excessive) fear response and subjective anxiety, and possibly enhancing fear extinction learning, may be beneficial during psychotherapy in PTSD. However, caution is warranted with regard to routine clinical OT application in the context of medication-enhanced psychotherapy for PTSD. In a recent pilot study, OT was administered prior to an exposure therapy session for arachnophobia (fear of spiders), investigating whether OT could augment exposure-based treatment response (Acheson et al, 2015). Although behavioral measures of fear were not altered, OT administration resulted in increased selfreported symptoms both at 1 week and 1 month follow-up, as well as decreased confidence in treatment and therapeutic alliance prior to the therapy session (Acheson et al, 2015).

To our knowledge, this is the first study investigating OT administration effects on amygdala subregion functional connectivity. Additionally, OT administration effects on resting-state functional connectivity have never been studied before in PTSD patients. Furthermore, male and female psychiatric patients have never been directly compared in one intranasal OT fMRI study on amygdala functional connectivity. However, several limitations need to be addressed. First, no causal inferences can be drawn based on the correlational functional connectivity analyses nor can we infer whether connections are inhibitory or excitatory. Although the vast majority of participants (ie, $>85 \%$ ) was able to let their mind wander during resting-state scanning, we cannot exclude that differences in content of thought between PTSD patients and trauma-exposed controls may have influenced our observed resting-state differences between PTSD patients and trauma-exposed controls. Future resting-state studies in PTSD patients should investigate the possible effects of content of thought on functional connectivity findings. Additionally, although our overall sample size was fairly large for a crossover fMRI study in psychiatric patients, it was relatively small to investigate possible sex differential effects of OT administration. Furthermore, we included a specific, highly traumatized sample of police officers, who experienced police-related traumatic events. This allowed us to not only control for the possible confounding effect of high trauma exposure and differences in types of traumatic experiences but also limits the generalizability of our findings to other PTSD patient samples and types of traumatic experiences. Additionally, we included female participants using hormonal contraceptives, which may have influenced basal OT levels and OT receptor affinity (Caldwell et al, 1994). However, female PTSD patients did not differ from female trauma-exposed regarding hormonal contraceptive use, indicating that the observed differential OT effects between these groups are unlikely to be explained by differences in hormonal contraceptive use.
Finally, we were unable to control for the phase of menstrual cycle our female participants were scanned in, as we aimed to minimize the time and hence differences in PTSD symptom severity between both scanning sessions. As extinction recall (Milad et al, 2006) was previously found to be influenced by phase of menstrual cycle, future studies on medicationenhanced psychotherapy for PTSD should take phase of menstrual cycle into account.

In conclusion, we showed that OT administration dampened subjective anxiety and nervousness in PTSD patients and that aberrant functional connectivity patterns in PTSD patients were normalized to similar levels as traumaexposed controls. This suggests that OT has the potential to diminish fear expression and enhance treatment response in PTSD patients. Our findings open the way for research investigating the therapeutic potential of OT administration in PTSD patients in more clinical settings, for example, during symptom provocation, and eventually during exposure-based therapy.

\section{FUNDING AND DISCLOSURE}

The study is supported by grants from ZonMw, the Netherlands organization for Health Research and Development (grant no. 91210041), and the Academic Medical Center Research Council (grant no. 40-00812-98-10041). All authors declare that they have no biomedical financial interests and no potential conflicts of interest.

\section{ACKNOWLEDGMENTS}

We thank all participants for their participation in this study. We also thank Renée Hutter, Gré Westerveld, Marthe Hoofwijk, and all other personnel of the PDC police outpatient clinic for their valuable help with recruitment of the PTSD patients. This study is registered in the Netherlands Trial Registry (name: 'The effect of oxytocin on brain processes in PTSD', number: NTR3516, http://www.trialreg ister.nl/trialreg/admin/rctview.asp?TC = 3516).

\section{REFERENCES}

Acheson DT, Feifel D, Kamenski M, Mckinney R, Risbrough VB (2015). Intranasal oxytocin administration prior to exposure therapy for arachnophobia impedes treatment response. Depress Anxiety 32: 400-407.

American Psychiatrics Association (2013). DSM-V, Diagnostic and Statistical Manual of Mental Disorders. American Psychiatric Publishing Inc.: Arlington, VA, USA.

Amunts K, Kedo O, Kindler M, Pieperhoff P, Mohlberg H, Shah NJ et al (2005). Cytoarchitectonic mapping of the human amygdala, hippocampal region and entorhinal cortex: intersubject variability and probability maps. Anat Embryol (Berl) 210: 343-352.

Bartz JA, Zaki J, Bolger N, Ochsner KN (2011). Social effects of oxytocin in humans: context and person matter. Trends Cogn Sci 15: 301-309.

Bertsch K, Gamer M, Schmidt B, Schmidinger I, Walther S, Kästel T et al (2013). Oxytocin and reduction of social threat hypersensitivity in women with borderline personality disorder. Am J Psychiatry 170: 1169-1177. 
Blake DD, Weathers FW, Nagy LM, Kaloupek DG, Gusman FD, Charney DS et al (1995). The development of a ClinicianAdministered PTSD Scale. J Trauma Stress 8: 75-90.

Bradley R, Greene J, Russ E, Dutra L, Westen D (2005). A multidimensional meta-analysis of psychotherapy for PTSD. Am J Psychiatry 162: 214-227.

Bremner JD, Bolus R, Mayer EA (2007). Psychometric properties of the Early Trauma Inventory-Self Report. J Nerv Ment Dis 195: 211-218.

Brown VM, Labar KS, Haswell CC, Gold AL, Beall SK, Van Voorhees E et al (2014). Altered resting-state functional connectivity of basolateral and centromedial amygdala complexes in posttraumatic stress disorder. Neuropsychopharmacology 39: 361-369.

Bryant RA, Felmingham K, Kemp A, Das P, Hughes G, Peduto A et al (2008a). Amygdala and ventral anterior cingulate activation predicts treatment response to cognitive behaviour therapy for post-traumatic stress disorder. Psychol Med 38: 555-561.

Bryant RA, Kemp AH, Felmingham KL, Liddell B, Olivieri G, Peduto A et al (2008b). Enhanced amygdala and medial prefrontal activation during nonconscious processing of fear in posttraumatic stress disorder: an fMRI study. Hum Brain Mapp 29: $517-523$.

Caldwell JD, Walker CH, Pedersen CA, Barakat AS, Mason GA (1994). Estrogen increases affinity of oxytocin receptors in the medial preoptic area-anterior hypothalamus. Peptides 15: 1079-1084.

Carlier I, Gersons B (1992). Development of a scale for traumatic incidents in police work. Psychiatr Fenn 23: 59-70.

Chao-Gan Y, Yu-Feng Z (2010). DPARSF: a MATLAB toolbox for 'pipeline' data analysis of resting-state fMRI. Front Syst Neurosci 4: 13.

de Kleine RA, Rothbaum BO, van Minnen A (2013). Pharmacological enhancement of exposure-based treatment in PTSD: a qualitative review. Eur J Psychotraumatol 4.

de Oliveira DCG, Zuardi AW, Graeff FG, Queiroz RHC, Crippa JAS (2012). Anxiolytic-like effect of oxytocin in the simulated public speaking test. J Psychopharmacol 26: 497-504.

de Vries G, Olff M (2009). The lifetime prevalence of traumatic events and posttraumatic stress disorder in the Netherlands. J Trauma Stress 22: 259-267.

Dodhia S, Hosanagar A, Fitzgerald DA, Labuschagne I, Wood AG, Nathan PJ et al (2014). Modulation of resting-state amygdala-frontal functional connectivity by oxytocin in generalized social anxiety disorder. Neuropsychopharmacology 39: 2061-2069.

Eckstein M, Becker B, Scheele D, Scholz C, Preckel K, Schlaepfer TE et al (2014). Oxytocin facilitates the extinction of conditioned fear in humans. Biol Psychiatry 78: 194-202.

Eickhoff SB, Stephan KE, Mohlberg H, Grefkes C, Fink GR, Amunts $\mathrm{K}$ et al (2005). A new SPM toolbox for combining probabilistic cytoarchitectonic maps and functional imaging data. Neuroimage 25: 1325-1335.

Fani N, Jovanovic T, Ely TD, Bradley B, Gutman D, Tone EB et al (2012). Neural correlates of attention bias to threat in posttraumatic stress disorder. Biol Psychol 90: 134-142.

Feifel D, Macdonald K, Nguyen A, Cobb P, Warlan H, Galangue B et al (2010). Adjunctive intranasal oxytocin reduces symptoms in schizophrenia patients. Biol Psychiatry 68: 678-680.

Felmingham K, Kemp A, Williams L, Das P, Hughes G, Peduto A et al (2007). Changes in anterior cingulate and amygdala after cognitive behavior therapy of posttraumatic stress disorder. Psychol Sci 18: 127-129.

First MB, Spitzer RL, Gibbon M, Williams JB. (2012). Structured Clinical Interview for DSM-IV Axis I Disorders (SCID-I)s, Clinician Version, Administration Booklet. American Psychiatric Publishing Inc.: Arlington, VA.
Foa EB, Keane TM, Friedman MJ, Cohen JA (2009). Effective Treatments for PTSD: Practice Guidelines from the International Society of Traumatic Stress Studies. Guilford Press: New York, NY, USA.

Hayes JP, Hayes SM, Mikedis AM (2012). Quantitative metaanalysis of neural activity in posttraumatic stress disorder. Biol Mood Anxiety Disord 2: 9.

Jovanovic T, Ressler KJ (2010). How the neurocircuitry and genetics of fear inhibition may inform our understanding of PTSD. Am J Psychiatry 167: 648-662.

Kirsch P, Esslinger C, Chen Q, Mier D, Lis S, Siddhanti S et al (2005). Oxytocin modulates neural circuitry for social cognition and fear in humans. J Neurosci 25: 11489-11493.

Koch SB, van Zuiden M, Nawijn L, Frijling JL, Veltman DJ, Olff M (2015). Intranasal oxytocin administration dampens amygdala reactivity towards emotional faces in male and female PTSD patients. Neuropsychopharmacology (doi:10.1038/npp.2015.299; e-pub ahead of print).

Koch SBJ, van Zuiden M, Nawijn L, Frijling JL, Veltman DJ, Olff M (2014). Intranasal oxytocin as strategy for medicationenhanced psychotherapy of PTSD: salience processing and fear inhibition processes. Psychoneuroendocrinology 40: 242-256.

Labuschagne I, Phan KL, Wood A, Angstadt M, Chua P, Heinrichs $\mathrm{M}$ et al (2010). Oxytocin attenuates amygdala reactivity to fear in generalized social anxiety disorder. Neuropsychopharmacology 35: 2403-2413.

LeDoux J (1998). Fear and the brain: where have we been, and where are we going? Biol Psychiatry 44: 1229-1238.

Li Y, Qin W, Jiang T, Zhang Y, Yu C (2012). Sex-dependent correlations between the personality dimension of harm avoidance and the resting-state functional connectivity of amygdala subregions. PLoS One 7: e35925.

Lischke A, Gamer M, Berger C, Grossmann A, Hauenstein K, Heinrichs $\mathrm{M}$ et al (2012). Oxytocin increases amygdala reactivity to threatening scenes in females. Psychoneuroendocrinology 37: 1431-1438.

Milad MR, Goldstein JM, Orr SP, Wedig MM, Klibanski A, Pitman RK et al (2006). Fear conditioning and extinction: influence of sex and menstrual cycle in healthy humans. Behav Neurosci 120: 1196-1203.

Milad MR, Wright CI, Orr SP, Pitman RK, Quirk GJ, Rauch SL (2007). Recall of fear extinction in humans activates the ventromedial prefrontal cortex and hippocampus in concert. Biol Psychiatry 62: 446-454.

Nawijn L, van Zuiden M, Koch SBJ, Frijling JL, Veltman DJ, Olff M (2016a). Intranasal oxytocin enhances neural processing of monetary reward and loss in post-traumatic stress disorder patients and traumatized controls. Psychoneuroendocrinology.

Nawijn L, van Zuiden M, Koch SBJ, Frijling JL, Veltman DJ, Olff M (2016b). Intranasal oxytocin increases insula responses to social reward in post-traumatic stress disorder.

Olff M, Frijling JL, Kubzansky LD, Bradley B, Ellenbogen MA, Cardoso C et al (2013). The role of oxytocin in social bonding, stress regulation and mental health: an update on the moderating effects of context and interindividual differences. Psychoneuroendocrinology 38: 1883-1894.

Olff M, Langeland W, Witteveen A, Denys D (2010). A psychobiological rationale for oxytocin in the treatment of posttraumatic stress disorder. CNS Spectr 15: 522-530.

Paloyelis Y, Doyle OM, Zelaya FO, Maltezos S, Williams SC, Fotopoulou A et al (2014). A spatiotemporal profile of in vivo cerebral blood flow changes following intranasal oxytocin in humans. Biol Psychiatry (doi:10.1016/j.biopsych.2014.10.005; e-pub ahead of print).

Patel R, Spreng RN, Shin LM, Girard TA (2012). Neurocircuitry models of posttraumatic stress disorder and beyond: a metaanalysis of functional neuroimaging studies. Neurosci Biobehav Rev 36: $2130-2142$ 
Rothbaum BO, Davis M (2003). Applying learning principles to the treatment of post-trauma reactions. Ann NY Acad Sci 1008: $112-121$

Roy AK, Shehzad Z, Margulies DS, Kelly AMC, Uddin LQ, Gotimer K et al (2009). Functional connectivity of the human amygdala using resting state fMRI. Neuroimage 45: 614-626.

Sheehan DV, Lecrubier Y, Sheehan KH, Amorim P, Janavs J, Weiller E et al (1998). The Mini-International Neuropsychiatric Interview (M.I.N.I.): the development and validation of a structured diagnostic psychiatric interview for DSM-IV and ICD-10. J Clin Psychiatry 59 Suppl 2: 22-33; quiz 34-57.

Sripada CS, Phan KL, Labuschagne I, Welsh R, Nathan PJ, Wood AG (2013). Oxytocin enhances resting-state connectivity between amygdala and medial frontal cortex. Int J Neuropsychopharmacol 16: 255-260.

Sripada RK, King AP, Garfinkel SN, Wang X, Sripada CS, Welsh RC et al (2012a). Altered resting-state amygdala functional connectivity in men with posttraumatic stress disorder. J Psychiatry Neurosci 37: 241-249.

Sripada RK, King AP, Welsh RC, Garfinkel SN, Wang X, Sripada CS et al (2012b). Neural dysregulation in posttraumatic stress disorder: evidence for disrupted equilibrium between salience and default mode brain networks. Psychosom Med 74: 904-911.

Stefanacci L, Amaral DG (2002). Some observations on cortical inputs to the macaque monkey amygdala: an anterograde tracing study. J Comp Neurol 451: 301-323.

Striepens N, Kendrick KM, Hanking V, Landgraf R, Wüllner U, Maier W et al (2013). Elevated cerebrospinal fluid and blood concentrations of oxytocin following its intranasal administration in humans. Sci Rep 3: 3440.

van Rooij SJH, Kennis M, Vink M, Geuze E (2015). Predicting treatment outcome in PTSD: a longitudinal functional MRI study on trauma-unrelated emotional processing. Neuropsychopharmacology (e-pub ahead of print).

Yan C-G, Cheung B, Kelly C, Colcombe S, Craddock RC, Di Martino A et al (2013). A comprehensive assessment of regional variation in the impact of head micromovements on functional connectomics. Neuroimage 76: 183-201.

Zoicas I, Slattery DA, Neumann ID (2014). Brain oxytocin in social fear conditioning and its extinction: involvement of the lateral septum. Neuropsychopharmacology 39: 3027-3035.

Supplementary Information accompanies the paper on the Neuropsychopharmacology website (http://www.nature.com/npp) 